\title{
On Polyhedra with Transitivity Properties
}

\author{
J. M. Wills
}

Mathematics Institute, University of Siegen, Hoelderlinstrasse 3, D-5900 Siegen, FRG

\begin{abstract}
In a remarkable paper [4] Grünbaum and Shephard stated that there is no polyhedron of genus $g>0$, such that its symmetry group acts transitively on its faces. If this condition is slightly weakened, one obtains some interesting polyhedra with face-transitivity (resp. vertex-transitivity).
\end{abstract}

In recent years several attempts have been made to find polyhedral analogues for the Platonic solids in the Euclidean 3-space $E^{3}$ (cf. $[1,4,5,7-14]$ ). By a polyhedron $P$ we mean the union of a finite number of plane polygons in $E^{3}$ which form a 2-manifold without boundary and without self-intersections, where, as usual, a plane polygon is a plane set bounded by finitely many line segments. If the plane polygons are disjoint except for their edges, they are called the faces of $P$. We require adjacent faces to be non-coplanar. The vertices and edges of $P$ are the vertices and edges of the faces of $P$. The genus of the underlying 2-manifold is the genus of $P$. A flag of $P$ is a triplet consisting of one vertex, one edge incident with this vertex, and one face containing this edge. If, for given $p \geq 3$, $q \geq 3$ all faces of $P$ are $p$-gons and all vertices $q$-valent, we say that $P$ is equivelar, i.e., has equal flags (cf. [7], [8] for convex faces). Equivelarity is a weak local analogue to the global property of flag-transitivity. In the following we always require that the polyhedra are equivelar. We denote an equivelar polyhedron of genus $g$ by $\{p, q ; g\}$ (cf. [7], [8]). For given $p, q, g$, nonisomorphic polyhedra $\{p, q ; g\}$ do exist.

One way to find close analogues to the Platonic solids is to consider polyhedra whose automorphism group is flag-transitive (cf. [9]-[11]). Another possibility which we consider here is to require only face- or vertex-transitivity under the symmetry group or another appropriate group of automorphisms.

Brehm's flat torus (cf. [1, p. 5], and [2, p. 438]) seems to be the first vertextransitive polyhedron of genus $g>0$ that was discovered; a model was shown at the 1978 Oberwolfach conference on convex bodies.

By a similar process Grünbaum and Shephard [4] found three highly symmetric 
equivelar polyhedra of genus 3,5, and 11 (and two nonequivelar polyhedra of genus 7 and 19) where the symmetry group acts transitively on their vertices. (The construction is given in the case of $g=5$, and it is not hard to see how the construction works for $g=7,11,19$. For $g=3$ one needs some additional conditions to avoid self-intersections. A construction for $g=3$, which seems to be easier than that in [4], is given in [11].)

In [4] Grünbaum and Shephard also stated that no polyhedra with analogous face-transitivity exist, thus underlining the exceptional property of their polyhedra. In [13] (compare also [12]) the author has considered weaker transitivity properties, allowing automorphism groups which contain the symmetry group as a subgroup of index 2 . This way one also obtains face-transitive polyhedra. In this paper (which might be considered as part II of [13]), we show that a stronger condition than that given in [13] (and therefore closer to that of Grünbaum and Shephard) yields face-transitive polyhedra.

Definition. An equivelar polyhedron with the rotation group of a Platonic solid is called a platonohedron if an automorphism group isomorphic to its full symmetry group acts transitively on its vertices or faces.

Remark 1. Every platonohedron is a Platonic manifold in the sense of [13]. These notions (and the notion "semi-Platonic" in [12]) only serve as simple keywords for the definitions. They underline the relations to the Platonic solids (realization in $E^{3}$, equivelarity, transitivities); no further philosophy is intended.

Remark 2. If one distorts the Platonic solids in a suitable way, one obtains platonohedra of genus 0 . This is trivial in the tetrahedral, octahedral, and cubic case. For the icosahedron and the dodecahedron this process is described in [13]. Clearly, the more interesting platonohedra are those of positive genus:

\section{Theorem.}

(a) There are at least five vertex-transitive and two face-transitive platonohedra with positive genus.

(b) There are at most finitely many combinatorial types of platonohedra.

Proof. (b) follows from simple combinatorial arguments, as given in [12], [13] for wider classes of polyhedra. The crucial point is (a), to show that there are four platonohedra (of genus 7 ) besides the $\{3,8 ; g\}, g=3,5,11$ of Grünbaum and Shephard, which clearly are platonohedra. First the $\{4,5 ; 7\}$ and $\{5,4 ; 7\}$ of $[12,13]$ are platonohedra, both having the complete octahedral symmetry group $S_{2} \times S_{4}$ and an isomorphic group acting transitively on the vertices of $\{4,5 ; 7\}$ (resp. on the faces of the "hexacis-octahedron" $\{5,4 ; 7\}$ ) (cf. [13]). Further, in Figs. 1 and 2 we show two remarkable platonohedra of genus 7 .

The $\{3,9 ; 7\}$ is constructed from two icosahedra; the interior one distorted such that the tetrahedral rotation group is preserved. If one deletes in an appropriate way eight faces of each icosahedron and joins the corresponding holes by triangular tubes, one obtains the $\{3,9 ; 7\}$; see Fig. 1 . 


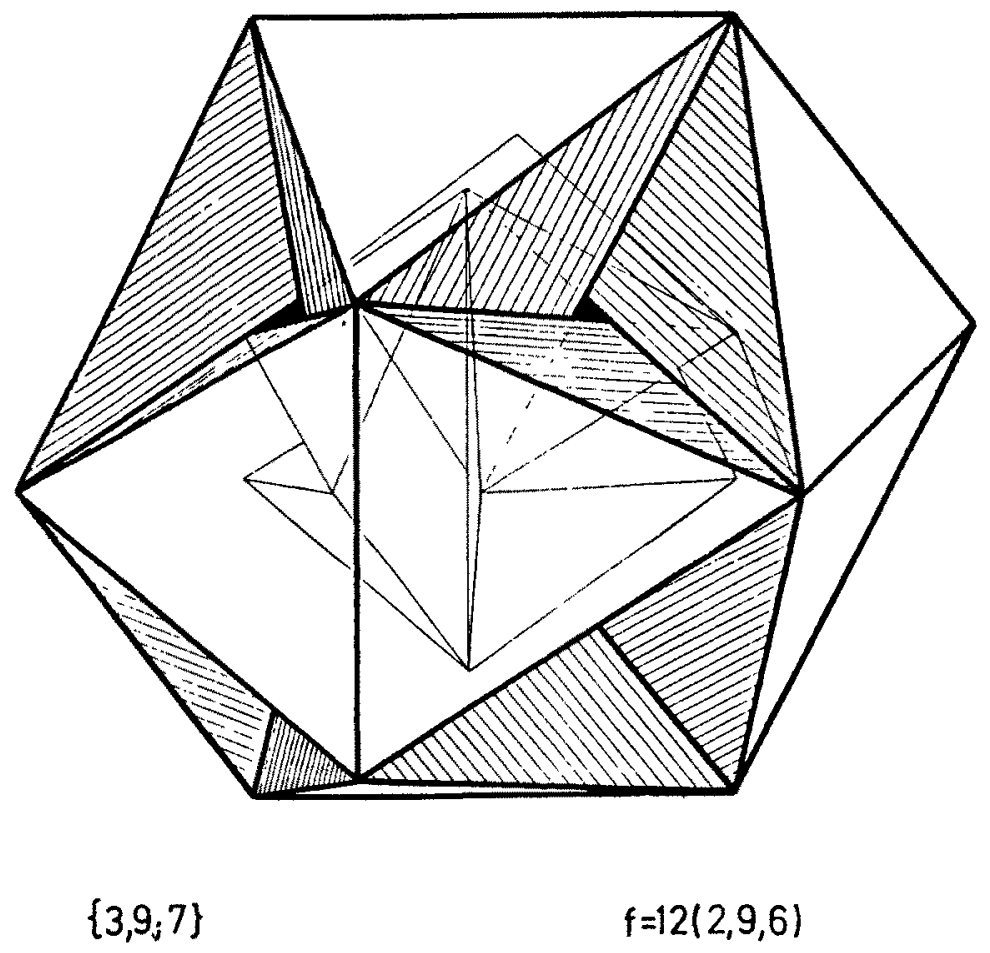

Fig. 1

The distorted icosahedron as well as the following distorted dodecahedron traces back to Jessen (cf. [6], compare also Grünbaum and Shephard [5]). Starting from a regular dodecahedron and a distorted dodecahedron (again with the tetrahedral symmetry group preserved) the "dual" process of taking an appropriate intersection of both solids gives the "disdodecahedron" $\{9,3 ; 7\}$ of Fig. 2 , which is in fact dual to the plyhedron of Fig. 1. A very similar process has been described in detail in [13].

By construction each of the 24 nonconvex 9-gons has a symmetry-axis and indeed the $\{9,3 ; 7\}$ and also the $\{3,9 ; 7\}$ are centrally symmetric with respect to their center.

So it is easy to see that they have the symmetry group $S_{2} \times A_{4}$, where $A_{4}$ is the tetrahedral rotation group. (We remark that $S_{2} \times A_{4}$, being a subgroup of the full octahedral and icosahedral symmetry group, is not isomorphic to the full tetrahedral symmetry group $S_{4}$.)

However, the symmetry group of $\{9,3 ; 7\}$ (resp. of $\{3,9 ; 7\}$ ) is not transitive on faces or vertices. But there is a subgroup of the automorphism group isomorphic to $S_{2} \times A_{4}$ which acts transitively on the vertices of $\{3,9 ; 7\}$ (resp. faces of $\{9,3 ; 7\}$ ). Here the factor $A_{4}$ is provided by the rotation group of the polyhedron. 


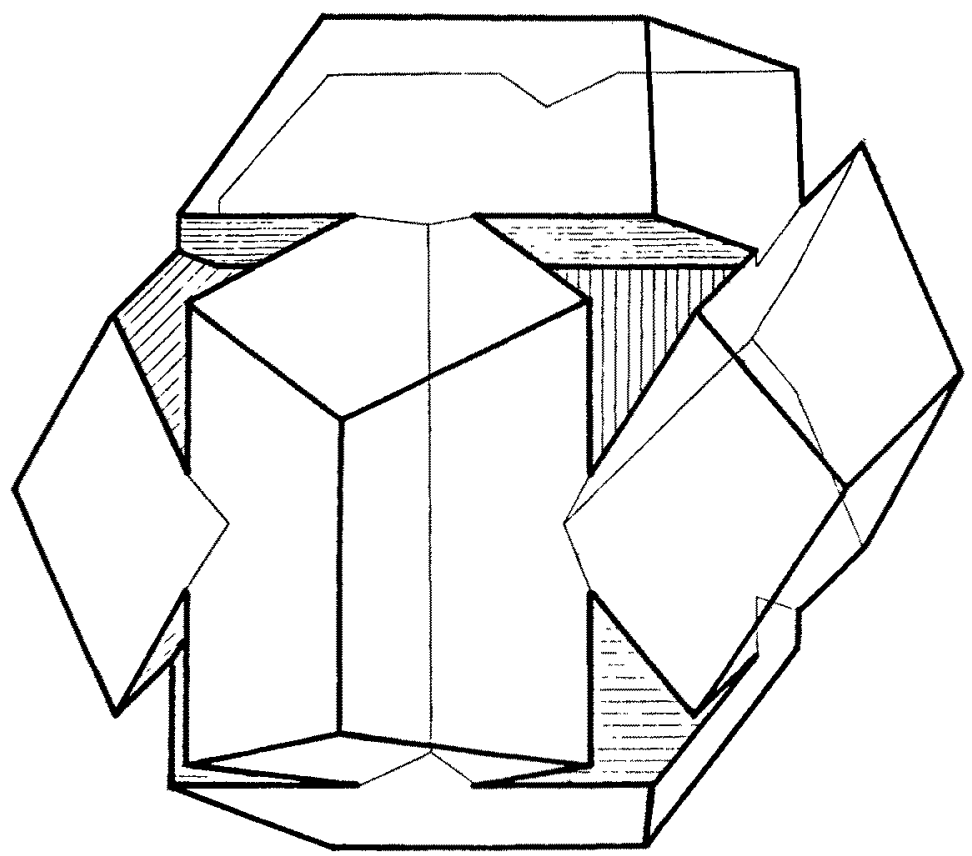

$\{9,3 ; 7\}$

$f=12(6,9,2)$

Fig. 2

The involution representing $S_{2}$ is given by an "inside-outside-symmetry" interchanging the roles of the outer vertices (resp. faces) and the corresponding inner vertices (resp. faces). This inside-outside-symmetry $\varphi$ is particularly evident for the polyhedron $\{3,9 ; 7\}$. Here the triangular faces of the outer icosahedron form six pairs connected as the quadrangular faces of the cuboctahedion. The same is true for the inner icosahedron, and the edges separating the faces in corresponding pairs of the inner and outer icosahedron are parallel. $\varphi$ just interchanges these edges, that is, maps the ends of each edge onto the corresponding ends of the parallel edge.

Now, observing that the six triangular faces surrounding a "tunnel" fit together like the faces of an octahedron with one pair of antipodal faces removed and that the effect of $\varphi$ on these faces is the same as the effect of the central inversion on the octahedron shows $\varphi$ is in fact an automorphism of the polyhedron.

With analogous arguments we obtain the corresponding result for the $\{9,3 ; 7\}$.

Concluding, we mention: (a) the $\{3,8 ; g\}$ are "chirohedra", i.e., they exist in chiral pairs; the other four platonohedra do not; (b) one obtains other $\{3,9 ; 7\}$, $\{9,3 ; 7\}$, nonisomorphic to those described before, if one rotates the inner icosahedron (resp. dodecahedron) in a suitable way about $\frac{1}{2} \pi$ (cf. [14, Fig. 10]). 


\section{Acknowledgments}

I would like to thank Egon Schulte and the referee for several helpful suggestions.

\section{References}

1. U. Brehm, Flacher torus, Tagungsberichte über konvexe Körper, No. 19, 5, Oberwolfach, 1978.

2. U. Brehm and $W$. Kïhnel, Smooth approximation of polyhedral surfaces regarding curvatures, Geom. Dedicata 12 (1982), 435-461.

3. H. S. M. Coxeter, Regular Polytopes, Dover, New York, 1973.

4. B. Grünbaum and G. Shephard, Polyhedra with transitivity properties, C.R. Math. Rep. Acad. Sci. Canada 6 (1984), 61-66.

5. B. Grünbaum and G. Shephard, Duality of polyhedra, Proceedings of the Conference on Shaping Space, Smith College, MA, 1984.

6. B. Jessen, Orthogonal icosahedron, Nordisk Mat. Tidskr. 15 (1967), 90-96.

7. P. McMullen, Ch. Schulz, and J. M. Wills, Equivelar polyhedral manifolds in $E^{3}$, Israel J. Math. 41 (1982), 331-346.

8. P. McMullen, Ch. Schulz, and J. M. Wills, Polyhedral 2-manifolds in $E^{3}$ with unusually large genus, Israel J. Math. 46 (1983), 127-144.

9. E. Schulte and J. M. Wills, A polyhedral realization of Felix Klein's map $\{3,7\}_{8}$ on a Riemannian manifold of genus 3, I. London Math. Soc, 32 (1985) 539-547.

10. E. Schulte and J. M. Wills, On Coxeter's regular skew polyhedra, Disc. Geom., to appear.

11. E. Schulte and J. M. Wills, Geometric realizations of Dyck's regular map $\{3,8\}_{6}$, Discrete Comput. Geom. 1 (1986), 141-154.

12. J. M. Wills, Semi-Platonic manifolds, in Convexity and Its Applications (P. M. Gruber and J. M. Wills, eds.), Birkhäuser, Basel, 1983.

13. J. M. Wills, Platonic manifolds, Mitt. Math. Sem. Giessen (Coxeter Festschrift) 163 (1984), 45-60.

14. J. M. Wills, Platonic manifolds and the flag diagram, Proceedings of the Conference on Shaping Space, Smith College, MA, 1984.

Received January 12, 1985. 